FECHA DE

PUBLICACIÓN:

$17-12-2021$

FECHA DE

ACEPTACIÓN:

02-11-2021

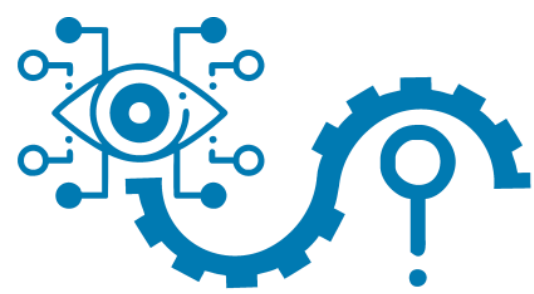

GnasisWisdam

\section{REVISTA DE INVESTIGACIÓN E INNOVACIÓN CIENTÍFICA Y TECNOLÓGICA}

Artículo original

Volumen 1, Número 3, Setiembre - Diciembre 2021

\title{
CALIDAD DEL AGUA POTABLE Y SU INFLUENCIA EN LA SALUD HUMANA
}

Drinking water quality and its influence on human health

\author{
Autores \\ Carlos, Dueñas Jurado ORCID \\ Universidad Nacional de Huancavelica \\ Lizangela, Hinojosa Yzarra ORCID \\ Universidad Nacional Autónoma de Huanta
}

\section{Resumen}

La sustancia esencial para la sobrevivencia de la vida en nuestro globo terráqueo es el agua, por lo que cada ser humano tiene el derecho de acceder a la buena calidad de este servicio, el cual es un indicador del desarrollo sostenible e influye en el campo de la salud ambiental. El presente estudio tuvo el objetivo de comprobar la influencia de la calidad de agua sobre las posibles enfermedades que pueda contraer el ser humano al consumir agua contaminada. Se desarrolló una revisión exhaustiva en diversas bases de datos como Web of Science, Scopus, PubMed, SciELO, Redalyc y Latindex, utilizando las palabras clave "Calidad del agua", "Parámetros cualitativos del agua", "Riesgos en la salud", "Agua contaminada", "Agua potable". Como resultado se puede indicar que los parámetros químicos como metales pesados son los de mayor relevancia y que mayores problemas ocasionan en la salud humana, así como también los microbiológicos que contaminan a través de bacterias sobre todo de origen fecal, ocasionando enfermedades gastrointestinales; mientras que, los parámetros físicos no tienen influencia directa.

Palabras clave: Agua contaminada, agua potable, parámetros cualitativos.

\begin{abstract}
The essential substance for the survival of life on our globe is water, so every human being has the right to have access to good quality of this service, which is an indicator of sustainable development and influences the field of environmental health. The present study had the objective of verifying the influence of water quality on the possible diseases that humans can contract when consuming contaminated water. An exhaustive review was carried out in various databases such as Web of Science, Scopus, PubMed, SciELO, Redalyc and Latindex, using the keywords "Water quality", "Qualitative parameters of water", "Health risks", "Contaminated water", "Drinking water". As a result, it can be indicated that chemical parameters such as heavy metals are the most relevant and cause the greatest problems for human health, as well as microbiological parameters that contaminate through bacteria, especially of fecal origin, causing gastrointestinal diseases, while physical parameters have no direct influence.
\end{abstract}

Keywords: Contaminated water, drinking water, qualitative parameters.

\section{Introducción}

La sustancia inherente a la vida en el planeta Tierra es el agua, por lo que cada persona debe disponer de este elemento de forma suficiente, segura y accesible, generando beneficios a la salud del ser humano. Esta calidad del agua es un indicador fundamental del desarrollo sostenible por lo cual abarca el campo de salud ambiental (Villena, 2018), analizándose para ello los parámetros microbiológicos, físicos y químicos; de tal manera que estos resultados se corroboren con los 


\section{GnasisWisdam}

catálogos de calidad del agua formulados por la Organización Mundial de la Salud (OMS, 2011) y los Estándares de la Calidad Ambiental - ECA, indicados en la normatividad nacional vigente, concordante con Bracho y Fernández (2012).

De acuerdo a lo manifestado por Ramos y Pinilla (2020), la provisión de agua insegura en zonas rurales, se relaciona con la deficiencia en la infraestructura de potabilización y la falta de control eficiente de las unidades de potabilización como son las actividades operativas rutinarias, de acuerdo al análisis microbiológico estos se relacionan con la existencia de bacterias como Escherichia Coli y Coliformes que habitan en el agua.

Del estudio realizado por Paniagua (2017), las cuencas de la región de Huancavelica es un potencial acuífero en el Perú; sin embargo, el ámbito geográfico presenta actividades mineras, por lo que de acuerdo al análisis físico químico microbiológico los cuerpos de agua como el rio Grande Sicra, Opamayo y la laguna de Pacococha presentan por encima de los niveles máximos permisibles en cadmio, arsénico, boro y plomo, el cual, de ser consumida genera un riesgo para la salud humana.

De la investigación realizada por Giraldo y Carvajal (2019), sobre la calidad de agua distribuida en el acueducto de Hondita Hojas Anchas - Colombia, se puede indicar que no tiene relación directa con la salud de la población; no obstante, el acueducto debe garantizar la calidad correspondiente ya que es más económico invertir en estrategias preventivas en comparación a las correctivas.

Salud y agua son dos aspectos primordiales que están estrechamente relacionados con la calidad de vida de la gente. Se entiende entonces que el agua hace parte del desarrollo humano y demás formas de vida existente. Villena (2018) indica a la calidad del agua como un valor ecológico, el cual es indispensable para la salud, y describe las características físicas químicas y microbiológicas, cuyos parámetros brinden las condiciones adecuadas para ser apta para consumo humano. El problema de la calidad del agua aún persiste en todos los países, sean desarrollados o sub desarrollados, la calidad inadecuada del agua influye directamente en las personas que dependen de la fuente de agua de la cual se suministran y acceden, esto incrementa los riesgos de contraer enfermedades mediante transmisión hídrica, de los cuales el cólera y la esquistosomiasis siguen siendo los más recurrentes, todo ello tomando en consideración lo vertido por la Organización de las Naciones Unidas (ONU, 2019). En los últimos años en el Perú, el agua ha sido un tema central en las perspectivas de estrategias políticas de desarrollo, pero a pesar de contar en todo el territorio con la estrategia sanitaria para el muestreo y vigilancia de calidad de agua, aún persiste el problema de aparición de enfermedades en los diversos grupos etarios, elevando los gastos en los servicios de salud, esto concordando con lo indicado por Ángel (2019). Para mejorar la calidad de agua en Huancavelica, se viene implementando la cloración como herramienta para la prevención de enfermedades diarreicas y parasitarias, aunque este problema aún se manifiesta en la anemia y desnutrición crónica infantil, de acuerdo a lo indicado por el Comité Técnico de Saneamiento Básico COTESABA (2017), siendo causa de esta mala calidad los sistemas de abastecimiento de agua para consumo humano que carecen de procesos de tratamiento, monitoreo y gestión para garantizar su 


\section{GnasisWisdam}

calidad apropiada. En consecuencia, la población consume agua contaminada, el cual es un riesgo para la salud humana. Frente a este problema se hace necesario analizar el grado de asociación de los parámetros de calidad de agua con el riesgo que tiene de contraer alguna enfermedad el ser humano. El presente estudio desarrolla una revisión exhaustiva de la influencia de la calidad de agua en la salud del ser humano, quien puede contraer enfermedades al consumir agua contaminada

\section{Materiales y métodos}

La presente investigación se enmarcó en la búsqueda exhaustiva de la información concerniente al tema, para lo cual se recurrió a las distintas bases de datos como SciELO, Redalyc, Latindex, Web of Science, Scopus, PubMed, entre otros, utilizando las palabras clave "Parámetros cualitativos de agua", "agua contaminada", "agua potable"; así como también se realizó una búsqueda exhaustiva de tesis de grado relacionados al tema, en los repositorios universitarios como los de la Universidad Nacional Daniel Alcides Carrión, Pontificia Universidad Javeriana, Universidad Nacional de la Amazonía Peruana, Universidad de Antioquia, Universidad de La Habana, Universidad Nacional Santiago Antúnez de Mayolo, Universidad Nacional de San Cristóbal de Huamanga y la Universidad Nacional de Ingeniería, utilizando el método hermenéutico para la interpretación de los textos y el método heurístico para la recopilación de información.

\section{Revisión de literatura \\ Parámetros de calidad de agua}

\section{Físicos}

Como su propio nombre lo indica se refiere a las características físicas del agua, siendo susceptibles a los sentidos (olfato, vista, gusto, tacto y oído), tienen directa incidencia sobre las condiciones estéticas y de aceptabilidad del agua, estos son las características organolépticas (color, olor y sabor), temperatura, turbidez, conductividad, solidos totales disueltos (Flores et al., 2019).

\section{Turbiedad}

Ocasionada por partículas que se encuentran en suspensión que influyen a simple vista en la trasparencia del agua, aunque no se conocen exactamente cómo influye en la salud humana, habiéndose demostrado que es muy importante en el proceso de eliminación de microrganismos patógenos, ya que las partículas que causan la turbiedad protegen en forma física a los microorganismos (m.o.) de la acción del desinfectante como puede ser el cloro, La turbiedad se mide mediante un turbidímetro cuyas unidades se denominan unidades nefelométricas de turbiedad (Giraldo y Carvajal, 2019).

\section{Temperatura}

Es uno de los parámetros de mayor importancia, ya que esta actúa en las reacciones químicas, al igual que en la velocidad de reacción, y es 


\section{GnasisWisdam}

indispensable para determinar la aptitud para ciertos usos del agua, este indicador influye en el comportamiento de otros indicadores de calidad de agua como el pH, conductividad eléctrica, déficit de oxigeno entre otras variables fisicoquímicos (Paniagua, 2017).

\section{Conductividad eléctrica}

Se refiere a la capacidad que tiene el agua para conducir la energía eléctrica esto por las sustancias disueltas ionizadas presentes, es lo opuesto al concepto de resistividad, siendo un indicador de mineralización del agua, cuyas unidades de medida comúnmente utilizadas son el Siemens/cm (S/cm), con una magnitud de $10^{\wedge}-6$, es el microSiemens/cm $(\mu \mathrm{S} / \mathrm{cm})$, o en $10^{\wedge}-3$, es el miliSiemens $/ \mathrm{cm} \quad(\mathrm{mS} / \mathrm{cm})$. La conductividad eléctrica es proporcional a los sólidos disueltos en el agua, de tal manera que a mayor concentración mayor conductividad (Olivera, 2019).

\section{Químicos}

Estos parámetros comprenden a los orgánicos, los inorgánicos y los gases; los orgánicos miden la cantidad de materia orgánica (MO) existentes en el agua, de esto se entiende que a mayor concentración de $\mathrm{MO}$ en el agua menor calidad del agua, entre ellos tenemos DQO (demanda química de oxígeno), DBO (demanda bioquímica del oxígeno), y en los inorgánicos los de mayor relevancia son el $\mathrm{pH}$ y la concentración de sales, mientras entre los gases presentes generalmente en las aguas naturales son el oxígeno, dióxido de carbono y nitrógeno, que son gases comunes en la atmósfera, mientras que los gases presentes en las aguas residuales son el amoniaco, sulfuro de hidrógeno y metano, los cuales proceden de la descomposición de la MO; mientras que, en las aguas desinfectadas o potabilizadas se puede encontrar el ozono y el cloro, según lo vertido por Flores et al. (2019). A continuación se detallan los más importantes:

\section{Oxígeno disuelto}

Generalmente proviene del aire y su presencia es muy importante en el agua, de tal manera que un nivel bajo de presencia de oxígeno en el agua puede ser indicativo que el agua está muy contaminada, esto debido a la alta carga de MO o una actividad bacteriana muy intensa, por ello consideramos a este parámetro como un indicador del nivel de contaminación. Cuando los niveles de oxígeno disuelto en el agua caen a $5 \mathrm{mg} / \mathrm{L}$, la vida acuática es puesta bajo presión, cuando los niveles de oxígeno están por debajo de 1 a $2 \mathrm{mg} / \mathrm{L}$ por unas pocas horas pueden morir grandes cantidades de peces (Olivera, 2019).

\section{PH}

Este indicador tiene mucha influencia en muchos de los fenómenos ocurrentes en el agua y en la infraestructura de las redes de distribución de agua; y es que se puede indicar que no tiene efectos directos sobre la salud humana; sin embargo, 


\section{GnasisWisdam}

puede incidir durante la coagulación y desinfección del agua (Giraldo y Carvajal, 2019).

\section{Cloro residual}

Es la sustancia más común utilizada en los sistemas de desinfección, el cual consiste en la extracción, eliminación o desactivación de m.o. patógenos existentes en la misma. La destrucción y/o desactivación de los m.o. significa el final de la reproducción y/o crecimiento de los m.o., de tal manera que si no son eliminados estos pueden causar enfermedades de transmisión hídrica (Olivera, 2019).

\section{Metales pesados}

Los metales que tienen mayor impacto en la salud humana son cadmio, mercurio, plomo y arsénico, generando riesgos para la salud humana según su concentración en el agua que se consume, también el impacto puede ser sobre la salud mediante exposición prolongada o por bio-acumulación, estos pueden ocasionar daños al organismo y/o pueden ser cancerígenos (Reyes et al., 2016).

\section{Biológicos y/o microbiológicos}

Las aguas de consumo humano de origen superficial se encuentran expuestas a una amplia gama de factores los cuales pueden alterar su calidad biológica y esto puede ocasionar cambios complejos o simples con distintos niveles de intensidad. La alteración se origina en eventos naturales o en actividades antrópicas, como el uso doméstico del agua y de igual manera en generación de aguas residuales, actividades agrícolas, mineras, industriales, entre otras. La contaminación fecal de las fuentes de aguas superficiales de consumo humano es uno de los problemas que más preocupa a los países subdesarrollados, los bioindicadores más comunes se basan en medir la presencia de m.o. como bacterias coliformes que producen contaminación fecal y los m.o. patógenos que producen el cólera (Flores et al., 2019).

\section{Coliformes totales}

Están conformadas por las bacterias Enterobacteriaceae, su característica principal es el de fermentar la lactosa, producir ácido y gas en un promedio de 48 horas a una temperatura de 30 a 37 grados centígrados (Olivera, 2019).

\section{Coliformes termotolerantes.}

De las investigaciones realizadas se sabe que la contaminación fecal del agua está muy relacionada con la transmisión hídrica de agentes patógenos, también se les denomina bacilos gram-negativos no esporulados, generalmente son de origen intestinal, el microorganismo principal es la Escherichia coli (Olivera, 2019).

\section{Parásitos}

Dentro de los parásitos patógenos los cuales se transmiten por el consumo de agua se encuentran dos grupos: helmintos y protozoos 


\section{GnasisWisdam}

a) Helmintos: son m.o. pluricelulares muy resistentes a los cambios de humedad, temperatura y pH; así mismo a la desinfección por el cloro, los cuales son causantes de altas tasas de morbilidad por consumo de aguas contaminadas, los huevos de helmintos son indicadores de la existencia de parásitos en el agua (Campos et al., 2018).

b) Protozoos: sus formas parasitarias pueden ser trofozoitos, quistes $\mathrm{u}$ ooquistes que son generalmente retenidos en los procesos de filtración de los sistemas de tratamiento de agua potable y algunos son resistentes a la cloración como los ooquistes. Son causantes de enfermedades diarreicas en los seres vivos que parasitan (Menocal y Caraballo, 2014).

\section{Riesgos en la salud humana}

El Reglamento de la Calidad de Agua del Ministerio de Salud del Perú, publicado en el año 2010, establece los límites máximos permisibles (LMP) de los indicadores de calidad de agua para consumo humano, por lo que con la finalidad de facilitar la interpretación adecuada del reglamento se pretende realizar la descripción de los riesgos que podrían ocasionar en la salud humana (Mora et al., 2017), estos son:

a) Turbiedad; valores superiores al límite máximo permisible 5.00 Unidades Nefelometricas de Turbidez - UNT, no generan riesgo para la salud; sin embargo, por su apariencia puede generar rechazo por parte de los consumidores, los valores muy altos de la turbiedad, exigen mayores dosis de cloro para desinfección, el cual concordamos con Guzmán et al. (2015).

b) Temperatura; es un parámetro influyente en las reacciones químicas y velocidad de reacción en el agua y en otros indicadores de calidad como, déficit del oxígeno,, conductividad eléctrica y $\mathrm{PH}$, Este parámetro está ligado al problema global del cambio climático, estudios realizados indican una asociación positiva entre las elevadas temperaturas y la transmisión de salmonella en el periodo 2002 al 2011 en los Estados Unidos, de igual manera indica una relación positiva entre la epidemia del cólera en Nepal en el año 2010 con las elevadas temperaturas, tal como indica (Malagón et al., 2017). .

c) Conductividad; valores entre 400 a 1000 $\mu \mathrm{S} / \mathrm{cm}$, no representa ningún riesgo para la salud; no obstante, esto indica una posible contaminación, valores superiores a los 1000 $\mu \mathrm{S} / \mathrm{cm}$ indica presencia de posibles contaminantes como intrusión salina, por lo que indica un riesgo alto para la salud humana, tiene efecto en parámetros como cloruros, magnesio, calcio, dureza, sulfato, potasio entre otros, por lo cual este parámetro depende de la dureza y temperatura del agua, estudios epidemiológicos indican una relación directa de la dureza del agua con Litiasis Renal y enfermedades cardiovasculares el cual concordamos con Solis et al. (2018). 


\section{GnasisWisdam}

d) $\mathrm{PH}$; valores entre 5.5 y 8.5 no repercute sobre la calidad del agua, valores inferiores a 4.00 pueden generar riesgo para la salud humana, tal como irritación en las mucosas y órganos internos, incluso pueden causar ulceración de acuerdo con (Pérez, 2016).

e) Cloro residual; valores superiores a $1.00 \mathrm{mg} / \mathrm{l}$ genera rechazo en la población, mientras los valores menores a $0.30 \mathrm{mg} / \mathrm{l}$ representa una posibilidad de contaminación microbiana, exposiciones prolongadas y cloración en mayor concentración pueden generar subproductos como Trihalometanos, los cuales son de actividad mutagénica y cancerígena, en acuerdo a lo manifestado por Olmedo (2008).

f) metales pesados, entre ellos tenemos el cadmio (Cd), quien al superar los LMP ocasionan daños en los riñones e hígado, su vida media es de 30 años, el Plomo $(\mathrm{Pb})$ a nivel de contaminante se distribuye en huesos y dientes ocasionando daños, el mercurio $(\mathrm{Hg})$, en su condición de toxicidad daña al sistema nervioso, y el arsénico (As), en exceso es cancerígeno y puede dañar a cualquier órgano del ser humano, en concordancia con lo aseverado por Reyes et al. (2016),

g) Coliformes fecales; valores superiores al límite máximo permisible indicado en el reglamento representa un riesgo alto para la salud humana, ya que ello es muestra de que el agua se encuentra contaminada, al consumirse puede ocasionar enfermedades gastrointestinales, de acuerdo a lo aseverado por Larrea et al. (2013).

h) Parásitos; son causantes de enfermedades diarreicas, incluso de la muerte de ancianos, niños y pacientes inmunocomprometidos, de acuerdo a la afirmación realizada por Ríos et al. (2017).

\section{Resultados}

Como resultado de la presente revisión bibliográfica, podemos indicar que los parámetros químicos como son los metales pesados como $\mathrm{Hg}, \mathrm{Cd}, \mathrm{Pb}$ y el $\mathrm{As}$ originan enfermedades que afectan tanto a órganos vitales como riñón e hígado, como al sistema nervioso, sistema cardiovascular, entre otros, en muchos de los casos son cancerígenos, mientras los parámetros microbiológicos como los son las bacterias de origen fecal y parásitos, son los que mayores problemas ocasionan en la salud humana, originando enfermedades gastrointestinales, sin embargo los parámetros físicos no tienen influencia directa, en la salud humana.

\section{Discusión de resultados}

Las actividad minera y el crecimiento de las grandes urbes acrecientan la contaminación del agua por metales pesados los cuales ocasionan problemas en la salud dañando a los órganos vitales como hígado, riñón, pulmones, corazón, sistema nervioso y otros tejidos vitales del ser humano, esta concordamos con Reyes et al. (2016), quienes aseveran que el problema de contaminación por metales pesados principalmente como $\mathrm{Cd}$, As, $\mathrm{Pb}$ y $\mathrm{Hg}$ son un problema a nivel global, los cuales comprometen severamente la salud de la población, esto a través del agua, incluso puede afectar 


\section{GnasisWisdam}

la cadena alimenticia de los ecosistemas, los LMP de los metales en el agua se encuentran bien establecidas en la actualidad. Los m.o. como virus, bacterias, hongos, protozoos y algunos parásitos son responsables de la contaminación microbiológica del agua, esto debido al crecimiento poblacional, actividad antropogénica y al cambio climático, ocasionando enfermedades sobre todo gastrointestinales, en concordancia con lo manifestado por Ríos et al. (2017) quienes manifiestan que el control de los m.o. patógenos en el agua depende de los estudios epidemiológicos de las instituciones involucrados en temas de salud, estos sobre todo están relacionados con las enfermedades diarreicas agudas; sin embargo indica que muchos de los patógenos son difíciles de detectar, los principales bio-indicadores establecidos a nivel mundial son los coliformes fecales, E. coli, y Enterococos; no obstante, a la fecha, con las nuevas investigaciones realizadas, se ha logrado identificar otros m.o. tales como Cryptosporidium spp, Estreptococos fecales, Pseudomonas spp. y Norovirus, quienes optimizan los resultados; en contraposición con Giraldo y Carvajal (2019), quienes indican que la calidad del agua suministrada a la población del acueducto La Hondita Hojas Anchas en Colombia no está relacionada con la salud de la población indicada.

\section{Conclusiones}

Es importante realizar el monitorio de la calidad de agua potable, en donde los parámetros microbiológicos, físicos y químicos que no deben superar los LMP establecidos en la normatividad nacional, de no ser así estos ocasionan una serie de enfermedades poniendo en riesgo la salud humana, los parámetros químicos a través de metales pesados y microbiológicos a través de bacterias sobre todo de origen fecal son los que más influyen en la salud humana.

\section{Referencias}

Ángel, G. (2019). Calidad del agua según la estrategia sanitaria de saneamiento básico de DIRESA en relación a los cinco primeros indicadores de morbilidad en el primer nivel de atención, Provincia de Pasco 2015. Tesis de maestría, Universidad Nacional Daniel Alcides Carrión, Cerro de Pasco, Perú. https://cutt.ly/SE2MfNy

Bracho, I. y Fernández, M. (2012). Evaluación de la calidad de las aguas para consumo humano en la comunidad venezolana de San Valentín, Maracaibo. Red de Revistas Científicas de América Latina, el Caribe, España y Portugal, 33 (3), 341 - 352. https://n9.cl/sy84e

Campos, M., Beltrán, M., Fuentes, N. y Moreno, G. (2018). Huevos de helmintos como indicadores de contaminación de origen fecal en aguas de riego agrícola, biosólidos, suelos y pastos. Revista del Instituto Nacional de Salud, 38 (1), 42 - 53. Pontificia Universidad Javeriana - Colombia. https://n9.cl/1wtey Comité Técnico de Saneamiento Básico, COTESABA. (2017). Acceso al agua apta para consumo humano Huancavelica 2015 - 2017. Biblioteca Nacional del Perú. https://n9.cl/ae0o6b

Flores, H., León, F., García, V. y Gilavert, G. (2019). Evaluación física, química y microbiológico de las aguas del río Nanay a orillas de la comunidad de Nina Rumi. Revista Ciencia y Tecnología, 15 (1), 113 - 122. 


\section{GnasisWisdam}

Universidad Nacional de la Amazonía Peruana Iquitos, Perú. https://n9.cl/dymtn

Giraldo, S. y Carvajal, V. (2019). Factores de riesgo de la calidad del agua para consumo humano y morbilidad sentida en usuarios del acueducto la Hondita Hojas Anchas, Guarne 2017. Tesis, Universidad de Antioquia, Medellín, Colombia. https://n9.cl/etifi

Guzmán, B., Nava, G. y Díaz, P. (2015). La calidad del agua para consumo humano y su asociación con la morbimortalidad en Colombia, 2008-2012. Revista Biomédica, 35 (2), 177-190. Instituto Nacional de Salud - Bogotá, Colombia. https://n9.cl/3t6ho

Larrea, J., Rojas, M., Álvarez, B., Rojas, N. y Heydrich, M. (2013). Bacterias indicadoras de contaminación fecal en la evaluación de la calidad de las aguas: revisión de la literatura. Revista CENIC Ciencias Biológicas, 44 (3), 24 - 34. Universidad de La Habana - Cuba. https://n9.cl/gywhm

Malagón, J., Garrote, C. y Castilla, P. (2017). Cambio climático y salud humana: una revisión desde la perspectiva colombiana. Revista Salud Uninorte, 33 (2), 224 - 241. Fundación Universidad del Norte Colombia. https://n9.cl/hk6kq

Menocal, L. y Caraballo, Y. (2014). Importancia de la vigilancia sanitaria de los parásitos en la calidad del agua, según su uso. Revista Cubana de Higiene y Epidemiología, 52 (2), 196-209. Instituto Nacional de Higiene - La Habana, Cuba. https://n9.cl/kdm2c

Mora, D., Orozco, J., Solís, Y., Rivera, P., Cambronero, D., Zúñiga, L. y García, J. (2017). Índice de riesgo de la calidad de agua para consumo humano en Costa Rica. Revista Tecnología en Marcha, 31 (3), 3 - 14. Costa Rica. https://n9.cl/sato4
Olivera, E. (2019). Influencia de la calidad de agua de consumo en la morbilidad por enfermedades de transmisión hídrica en la población infantil del distrito de Cátac-Recuay-Ancash durante el año 2016. Tesis de doctorado, Universidad Nacional Santiago Antúnez de Mayolo, Ancash, Perú. https://n9.cl/zpvoq

Olmedo, T. (2008). Subproductos de la desinfección del agua por el empleo de compuestos de cloro. Efectos sobre la salud. Revista Higiene y Sanidad Ambiental. https://n9.cl/6y6w0

Organización Mundial de la Salud, OMS. (2011). Guías para la calidad del agua de consumo humano. Organización Mundial de la Salud. https://n9.cl/ebku

Organización de las Naciones Unidas, ONU. (2019). Informe mundial de las Naciones Unidas sobre el desarrollo de los recursos hídricos 2019. UNESCO. https://n9.cl/ylqp7

Paniagua, O. (2017). Evaluación de la calidad del agua para consumo humano y fines agrícolas en las cuencas de la Región Huancavelica. Tesis, Universidad Nacional de San Cristóbal de Huamanga, Ayacucho, Perú. https://n9.cl/u823m

Pérez, E. (2016). Control de calidad en aguas para consumo humano en la región occidental de Costa Rica. Revista Tecnología en Marcha, 8 (1), 335 - 342. España. https://n9.cl/0p1ik

Ramos, Y. y Pinilla, M. (2020). Calidad de agua para consumo humano en sistema de abastecimiento rurales en Boyacá, Colombia. Un análisis infraestructural. Revista EIA, 17 (34), 1 - 15. Universidad de los Andes - Colombia. https://n9.cl/ghgp6 


\section{GnasisWisdam}

Reyes, Y., Vergara, I., Torres, O., Diaz, M. y Gonzales,

E. (2016). Contaminación por metales pesados: implicaciones en salud, ambiente y seguridad alimentaria. Revista Ingeniería, Investigación y Desarrollo, 16 (2), 66 - 77. Colombia. https://n9.cl/zdoz

Ríos, S., Agudelo, R. y Gutiérrez, L. (2017). Patógenos e indicadores microbiológicos de calidad del agua para consumo humano. Revista Fac. Nac. Salud Pública, 35 (2), 236 - 247. Universidad de Antioquia - Colombia. https://n9.cl/pgzc

Solís, Y., Zúñiga, L. y Mora, D. (2018). La conductividad como parámetro predictivo de la dureza del agua en pozos y nacientes de Costa Rica. Revista Tecnología en Marcha, 31 (1), 35 - 46. Costa Rica. https://n9.cl/3y64u

Villena, J. (2018). Calidad del agua y desarrollo sostenible. Revista Perú Med Exp Salud Publica, 35 (2), 304 - 308. Universidad Nacional de Ingeniería Lima, Perú. https://n9.cl/uizt0 